\title{
MMF for Lupus Nephritis as a Possible First Aid Block
}

See Cross et al., pp. c92-c100

\author{
Michio Nagata ${ }^{a}$ Koichi Hirayama ${ }^{b}$
}

Departments of ${ }^{\mathrm{a}}$ Molecular Pathology and ${ }^{\mathrm{b}}$ Nephrology, Graduate School of Comprehensive Human Sciences, University of Tsukuba, Tsukuba, Japan

Despite a significant advance in the immunosuppressive regimen, the control of lupus nephritis (LN) patients is often difficult and results in approximately $30-50 \%$ of patients developing chronic renal failure [1]. The optimal treatment for $\mathrm{LN}$ requires a high rate of remission induction and a long-standing suppression of disease activity without serious adverse effects. Cyclophosphamide (CYC) has been most used for severe LN. However, it occasionally shows severe adverse effects, including gonadal toxicity, hemorrhagic cystitis, infections and carcinogenesis. An additional serious problem is that approximately $15 \%$ of $\mathrm{LN}$ patients are resistant to CYC [1] and have a high incidence of renal relapse after a successful induction of CYC.

Mycophenolate mofetil (MMF) is a brand new immunosuppressant that selectively inhibits lymphocytes proliferation and has shown important results in preventing solid organ transplant rejection. Its distinct merit is fewer adverse effects compared to CYC. MMF treatment for LN was first reported in 1998 [2], when a favorable response was demonstrated in 2 cases of LN patients with nephrotic-range proteinuria. There have been many uncontrolled studies or case presentations reporting that MMF is occasionally effective on active $\mathrm{LN}$ including those who are refractory to CYC [3-7]. Insignificant adverse effects and a requirement for fewer steroids are benefits suggested by some studies. The majority of these reports included patients in various stages of the disease or with differing disease activity. It has not been determined which aspects of LN MMF specifically benefits.

The report in this issue of Nephron Clinical Practice by Cross et al. [8] is an open-labeled study testing MMF combined with prednisolone for remission induction in 24 patients who have active class III, IV or V nephritis. The merit of this study is focused on remission induction without previous exposure to CYC or MMF. Although the active and the chronic state of kidney histology are not described, it seems likely that patients in this study have predominant active LN. The authors found that 20/24 cases revealed a complete renal remission within 6 months as estimated by the BILAG Index. This was accompanied by improved clinical parameters, i.e., complement levels and circulating anti-double-stranded DNA antibodies. Among 18 adverse events, 10 were non-serious infections in the upper respiratory tract. One patient suffered from legionella pneumonia under a renal remission. The authors state that MMP with prednisolone was effective in remission induction in severe $\mathrm{LN}$ and was well tolerated. A previous study of MMF on RCT for LN by Chan et al. [9] indicated that a 12-month treatment with MMF and prednisolone showed a comparative efficacy with 6-month treatment of CYC and prednisolone. Although the present study is based on a non-RCT study in a small number of patients followed for a short term, this is an interesting finding suggesting that MMF with prednisolone could possibly be a first-line use for an active LN.

\section{KARGER \\ Fax +4161306 1234 E-Mail karger@karger.ch} www.karger.com (c) 2005 S. Karger AG, Basel $1660-2110 / 05 / 1003-0101 \$ 22.00 / 0$

Accessible online at: www.karger.com/nec
Prof. Michio Nagata

Department of Pathology, Institute of Basic Medical Sciences

University of Tsukuba, Tsukuba 305-8575 (Japan)

Tel. +81298533171, Fax +81298533938

E-Mailnagatam@md.tsukuba.ac.jp 
Most studies have reported MMF efficacy on admixtures of various LN histology or disease activities. Although it is generally agreed that MMF has an effect on proliferative lesions and on membranous LN [10], MMF has occasionally failed to stop LN. Histopathological examinations reveal that MMF ameliorates active inflammatory lesions including cell proliferation and inflammatory infiltrates, but efficacy is limited in chronic fibrotic lesions [11]. It is likely that MMF with prednisolone is effective in initial acute lesions of $\mathrm{LN}$ as reported by Cross et al. [8]. Since recurrent or smoldering active inflammatory foci promote chronic renal pathology in LN, remission induction by MMF suppressing early active lesions is of value. It is better to have a therapeutic option for $\mathrm{LN}$ determined by histological activity. Further studies need to be designed as multicenter RCTs based on the new LN glomerulonephritis classification [12], which consider activity and chronicity information.

The maintenance of remission and the inhibition of relapse are also hurdles in LN treatment. Frequently, the maintenance of immunosuppression with azathioprine (AZ) and prednisolone cause a recurrence of $\mathrm{LN}$ which needs CYC treatment. In this context, Bijl et al. [13] monitored antibodies against dsDNA in LN patients. They found that MMF prevented a clinical relapse predicted by the rise of dsDNA. Some reports also noted that a renal relapse occurred after MMF was switched to AZ. By contrast, recent experience shows that MMF-treated patients have a greater incidence of renal relapse compared with CYC/AZ [1]. A future aspect will include long-running maintenance of $\mathrm{LN}$ by MMF with minimal adverse effects. For this aim, the metabolic pathway of MMF in $\mathrm{LN}$ and an adequate blood monitoring needs to be established. A recent report by Haidinger et al. [14] measured mycophenolate acid and MPA-glucuronide by HPLC in autoimmune disease (AID) and renal transplant recipients (RTX). They found that mean MPA levels at $12 \mathrm{~h}$ were higher in AID than RTX, whereas peak levels were comparable in both. In addition, inter-individual variability of pharmacokinetic parameters was found. MPA, the metabolite of MMF, is known to bind tightly to protein and is excreted predominantly in urine. Thus, nephrotic or uremic states may alter the blood levels of free fractions. Future efforts are needed to establish safety, effective dosage and a monitoring system of MMF in AID patients while considering individual conditions.

Does MMF have an effect on LN of any genetic background? Racial differences in the progression of $\mathrm{LN}$ is a distinct issue for MMF treatment. MMF is well recognized in western communities and a majority of subjects treated by MMF are patients among Caucasians or Chinese. LN patients among African-Americans are progressive and are resistant to CYC pulse therapy. Thus, it may be possible that modified MMF treatment improves this outcome in a particular population or MMF might be an insufficient regimen for some ethnic LN patients. Further study should include control trials of MMF and CYC in high-risk groups of different ethnic populations.

Generally, medical insurance does not cover MMF treatment for LN. A long-term, large-scale RCT study and/or a strong petition on this potentially promising drug is now needed for LN patients.

\section{References}

1 Mok CC, Lai KN: Mycophenolate mofetil in lupus glomerulonephritis. Am J Kidney Dis 2002;40:447-457.

2 Briggs WA, Choi MJ, Scheel PJ Jr: Successful mycophenolate mofetil treatment of glomerular disease. Am J Kidney Dis 1998;31:213217.

3 Dooley MA, Cosio FG, Nachman PH, et al: Mycophenolate mofetil therapy in lupus nephritis: Clinical observations. J Am Soc Nephrol 1999;10:833-839.

4 Gaubitz M, Schorat A, Schotte H, et al: Mycophenolate mofetil for the treatment of systemic lupus erythematosus: An open pilot trial. Lupus 1999;8:731-736.

5 Kingdon EJ, McLean AG, Psimenou E, et al: The safety and efficacy of MMF in lupus nephritis: A pilot study. Lupus 2001;10:606611
6 Li L, Wang H, Lin S, et al: Mycophenolate mofetil treatment for diffuse proliferative lupus nephritis: A multicenter clinical trial in China. Zhonghua Nei Ke Za Zhi 2002;41:476479.

7 Karim MY, Alba P, Cuadrado MJ, et al: Mycophenolate mofetil for systemic lupus erythematosus refractory to other immunosuppressive agents. Rheumatology (Oxford) 2002;41: 876-882.

8 Cross J, Dwomoa A, Andrew P, et al: Mycophenolate mofetil for remission induction in severe lupus nephritis. Nephron Clin Pract 2005;100:c92-c100.

9 Chan TM, Li FK, Tang CS, et al: Efficacy of mycophenolate mofetil in patients with diffuse proliferative lupus nephritis. Hong KongGuangzhou Nephrology Study Group. N Engl J Med 2000;343:1156-1162.
10 Spetie DN, Tang Y, Rovin BH, et al: Mycophenolate therapy of SLE membranous nephropathy. Kidney Int 2004;66:2411-2415.

11 Ding L, Zhao M, Zou W, et al: Mycophenolate mofetil combined with prednisone for diffuse proliferative lupus nephritis: A histopathological study. Lupus 2004;13:113-118.

12 Weening JJ, D'Agati VD, Schwartz MM, et al: The classification of glomerulonephritis in systemic lupus erythematosus revisited. J Am Soc Nephrol 2004;15:241-250.

13 Bijl M, Horst G, Bootsma H, et al: Mycophenolate mofetil prevents a clinical relapse in patients with systemic lupus erythematosus at risk. Ann Rheum Dis 2003;62:534-539.

14 Neumann I, Haidinger M, Jager H, et al: Pharmacokinetics of mycophenolate mofetil in patients with autoimmune diseases compared renal transplant recipients. J Am Soc Nephrol 2003; 14:721-727. 